\title{
Ensuring safe sedation during gastroendoscopy
}

\author{
Eng Kiong Teo ${ }^{1}$ MRCP (UK), FRCP (Edin), FAMS
}

Gastrointestinal (GI) endoscopy started in Singapore in 1968 with diagnostic endoscopic gastric examination, using flexible fibre-optics GI endoscopy. Fibre-optic flexible sigmoidoscopy and colonoscopy were introduced later. Most of these procedures were performed then without sedation. The patients needed to cooperate with the endoscopists and inability to complete the endoscopic examination was common then due to intolerability of the procedure. By the 1980s, therapeutic GI endoscopy was introduced and subsequently practised as a standard of care. Since then, we have seen an expansion in the scope of therapeutic endoscopic procedures. With the increasing complexity of endoscopic procedures requiring a longer procedure time, moderate sedation during GI endoscopy becomes an inherent component for both patient comfort and patient safety as well as procedural success. Although some patients may opt for GI endoscopy without sedation, the vast majority of GI endoscopic procedures are currently performed under moderate sedation.

The training to administer moderate sedation is incorporated into the training curriculum of GI endoscopy. Trainees are taught the agents used to induce moderate sedation and the medications needed to reverse the sedation when needed. Common agents to induce moderate sedation are benzodiazepines and opiates used either singly or in combination. Endoscopists are also taught to assess and recognise the risks of moderate sedation. These include hypoventilation and haemodynamic instability. Although the administration of moderate sedation is an integral component of endoscopic training, there was no defined module and no distinct certification for privileges to administer moderate sedation until the 2000 s. In the early 2000s, Singapore healthcare system embarked on a journey of improvement and accreditation. The Joint Commission International (JCI) accreditation system was selected by many private and public healthcare institutions. Applying the standards of JCI, endoscopists (and others who perform moderate sedation) must show proof of competency for both administration of the agents, the use of reversal agents, and the ability to administer advanced airway and cardiopulmonary life support. Since then, endoscopists performing endoscopy under moderate sedation need to be certified at regular intervals to ensure the currency of their knowledge and skills in administration of moderate sedation.

Apart from the endoscopist, moderate sedation can be administered by the anaesthetist as well. In fact, the administration and monitoring of patients under moderate sedation is part of the curriculum for the training of anaesthesiology. Although the risk for mortality and other adverse events are less when compared to general anaesthesia, there is still a definite risk of over-sedation leading to hypoventilation and hypoxaemia. In addition, some of the agents used in moderate sedation can cause hypotension or bradycardia, leading to increased risk of stroke and acute myocardial infarction especially in patients with significant relevant comorbidities. As a result of adverse outcomes due to sedation, the Ministry of Health in Singapore commissioned the Academy of Medicine, Singapore (AMS) to provide guidelines on safe sedation practice for non-anaesthesiologists in medical clinics, stand-alone ambulatory surgical centres and stand-alone endoscopy suites in Singapore. This guideline was published on 19 May 2014 and recently updated in July 2021. This guideline formed the basis of many of our current practices in the administration of moderate sedation. ${ }^{1}$ The guideline outlines the need for pre-sedation assessment and to consider the engagement of an anaesthesiologist should the risk be high and/or the procedure is expected to be prolonged. The American Society of Anesthesiologists (ASA) score was recommended and airway assessment was also included so as to anticipate difficulty in securing definitive airway should the need arise. These pre-sedation assessments aimed at detecting patients with the highest risk for moderate sedation so that additional resources, e.g. an anaesthetist, can be channelled to these patients. For the patients without high risks for moderate sedation, the endoscopist and the endoscopic nurse, trained in the administration and monitoring of moderate sedation, will suffice. This is to ensure that the cost of endoscopic examination remained affordable while balancing the risk of adverse events.

\footnotetext{
${ }^{1}$ Department of Medicine, Sengkang General Hospital, Singapore

Correspondence: Prof Eng Kiong Teo, Department of Medicine, Sengkang General Hospital, 110 Sengkang East Way, Singapore 544886.

Email: teo.eng.kiong@singhealth.com.sg
} 
The guideline on moderate sedation published by AMS in 2014 specifically mentioned the use of propofol separately from other agents used for moderate sedation. The 2014 guideline recommended that propofol must be administered by an anaesthesiologist. This is because the experience with the use of propofol then for endoscopic procedures was limited and there was no reversal agent if a patient is over-sedated with propofol. In addition, propofol has a narrow therapeutic window, magnifying the risk of over-sedation and hypoventilation. However, propofol has significant pharmacological advantages over benzodiazepines. Unlike benzodiazepines, propofol is short-acting and does not have active metabolites that prolong the sedative effects after the endoscopic examination. Patient who received propofol for sedation recover more rapidly compared to those who received benzodiazepines. This study also showed that a nurse as a trained sedationist can effectively and safely administer the propofol and monitor the patient adequately. ${ }^{2}$ With these data on propofol, ${ }^{3-6}$ endoscopists in Singapore have also started using propofol as an agent for moderate sedation. These procedures are performed in endoscopy suites in the hospital setting where appropriate facilities and adequate manpower are available. The experience by many local endoscopists in Singapore mirrors that described in the reports in Western endoscopic centres.

In 2021, AMS revised the guidelines for sedation, and sedation for GI endoscopy was discussed separately. A workgroup consisting of representatives from the College of Anaesthesiologists, College of Surgeons and the Chapter of Gastroenterologists came together to review the available information and developed the guideline for sedation for GI endoscopy in the hospital setting. Current evidence was reviewed and were graded for the recommendations. This guideline seeks to update the community of endoscopists on current practices, with the primary aim of patient safety and secondary aim of providing a sustainable system without significant compromise on patient safety. This guideline covers multiple areas including consent, training requirements, agents available and the knowledge required to use these agents, use of reversal agents, monitoring of patients, and resuscitative competencies should the patient develop ventilatory or haemodynamic instability. ${ }^{7}$

It is the aim of AMS to offer professional guidance so that specialists can provide care with confidence that they are within international standards. This guideline on sedation in GI procedures is one such initiative. AMS as a professional body will continue to provide guidance so that Singapore can maintain its leadership in standards of medical care in the Asia-Pacific region.

\section{REFERENCES}

1. Guidelines on Safe Sedation Practice for Non-Anaesthesiologists in Medical \& Dental Clinics, Stand-Alone Ambulatory Surgical Centres, and Stand-Alone Endoscopy Suites in Singapore, July 2021. Available at: https://www.ams.edu.sg/view-pdf. aspx ?file $=$ media $\% 5$ C6241 fi 759.pdf\&ofile $=09$ July $21+$ Updat ed + Guidance + on + Safe + Sedation+Practice.pdf. Accessed on 4 January 2022.

2. Sipe BW, Rex DK, Latinovich D, et al. Propofol vs Midazolam/ meperidine for outpatient colonoscopy: Administration by nurses supervised by endoscopists. Gastrointest Endosc 2002;55:815-25.

3. Qadeer MA, Vargo JJ, Khandwala F, et al. Propofol versus traditional sedative agents for gastrointestinal endoscopy: a metaanalysis. Clin Gastroenterol Hepatol 2005;3:1049-56.

4. Dossa F, Medeiros B, Keng C, et al. Propofol versus midazolam with or without short-acting opioids for sedation in colonoscopy: a systematic review and meta-analysis of safety, satisfaction, and efficiency outcomes. Gastrointest Endosc 2020;91:1015-26.e7.

5. Ferreira AO, Torres J, Barjas E, et al. Non-anesthesiologist administration of propofol sedation for colonoscopy is safe in low risk patients: results of a noninferiority randomized controlled trial. Endoscopy 2016;48:747-53.

6. Daza JF, Tan CM, Fielding RJ, et al. Propofol administration by endoscopists versus anesthesiologists in gastrointestinal endoscopy: a systematic review and meta-analysis of patient safety outcomes. Can J Surg 2018;61:226-36.

7. Ang TL, Seet E, Goh YC, et al. Academy of Medicine, Singapore Clinical Guideline on the Use of Sedation by Non-anaesthesiologists during Gastrointestinal Endoscopy in the Hospital Setting. Ann Acad Med Singap 2022;51:24-39. 